vague and indeterminate; it virtually asserts that, if two infinite magnitudes be equal, the addition of any finite magnitude to either of them will destroy the equality.

In closing this note I desire to guard against the danger of leaving a false impression. The mere correctness of the Lucretian concept of infinity by no means accounts for the immense rôle of the concept in the author's work. The secret lies in the fact that the concept so powerfully stimulated the imagination of a great thinker and poet as to cause him to express and to preserve in immortal form a body of ideas which he had acquired from the then still extant works of Epicurus and which after the long lapse of centuries are found to be among the most fruitful scientific ideas of our time.

Columbia UnIVersity, December, 1917.

\title{
ON THE INVARIANT NET OF CUBICS IN THE STEINERIAN TRANSFORMATION.
}

BY PROFESSOR ARNOLD EMCH.

(Read before the American Mathematical Society September 4, 1917.)

1. By Steinerian transformation* we understand an involutorial quadratic Cremona transformation, defined as the one-to-one correspondence between the points of a plane (with the exception of the points of a certain trilateral) and the points of concurrence of their polars with respect to the conics of a pencil. If we use the base points $A_{1}, A_{2}, A_{3}, E$ of the pencil as the vertices and the unit point of a system of projective coordinates, the Steinerian transformation may easily be established in the form

$$
\begin{aligned}
& \rho x_{1}^{\prime}=x_{1}\left(x_{2}+x_{3}-x_{1}\right), \\
& \rho x_{2}^{\prime}=x_{2}\left(x_{3}+x_{1}-x_{2}\right), \\
& \rho x_{3}{ }^{\prime}=x_{3}\left(x_{1}+x_{2}-x_{3}\right) .
\end{aligned}
$$

The base points $A_{1}(1,0,0) ; A_{2}(0,1,0) ; A_{3}(0,0,1) ; E(1,1$,

* See Annals of Mathematics, vol. 14 (1912), pp. 57-71. 
1) of the pencil are invariant under the transformation, while to the diagonal points $E_{1}(0,1,1) ; E_{2}(1,0,1) ; E_{3}(1,1,0)$ correspond the opposite diagonals of the diagonal triangle of the base point quadrangle. The net of cubics through the seven points $A_{1}, A_{2}, A_{3}, E, E_{1}, E_{2}, E_{3}$ may be represented in the form

$$
a_{1} x_{2} x_{3}\left(x_{2}-x_{3}\right)+a_{2} x_{3} x_{1}\left(x_{3}-x_{1}\right)+a_{3} x_{1} x_{2}\left(x_{1}-x_{2}\right)=0,
$$

and is invariant under the transformation (1). It is not difficult to establish the

THEOREM: For every set of values $\left(a_{1}: a_{2}: a_{3}\right)$ equation (2) represents a cubic for which the coordinate triangle $A_{1} A_{2} A_{3}$ and the unit point $E$ form a Steinerian quadruple, such that the tangents to the cubic at these points meet in a point $P$ of the cubic whose coordinates are precisely $a_{1}, a_{2}, a_{3}$.

Conversely, when from a point $P$ of an elliptic cubic we draw the four tangents to the cubic with the points of tangency $A_{1}, A_{2}$, $A_{3}, E$, and we choose these as the vertices and unit point of $a$ projective coordinate triangle, in which the coordinates of $P$ are $a_{1}, a_{2}, a_{3}$, then the equation of the cubic may be written in the form (2).

2. It is known that on every straight line $l$ in a general position there are two points $P$ and $P^{\prime}$ only which correspond to each other in the Steinerian transformation. They are obtained as the points of intersection of $l$ and the corresponding conic $K$ through the diagonal points, or also as the double points of the involution cut out on $l$ by the conics of the pencil through the base points. The cubic may be generated by the pencil of lines through $P$ and the projective pencil of corresponding conics through $E_{1}, E_{2}, E_{3}$, and $P^{\prime}$. It may also be considered as the locus of pairs of corresponding points on the rays of the pencil through $P$. For the sake of brevity the point of concurrence of the tangents to a cubic at the points of an inscribed Steinerian quadruple shall be called an $S$-point of the cubic. Two cubics $C_{P}$ and $C_{Q}$ of the net intersect in two points $X$ and $X^{\prime}$. As both are invariant under the transformation, the corresponding point of $X$ must lie on $C_{P}$ as well as on $C_{Q}$, and must therefore coincide with $X^{\prime}$. Hence the

TheOREM: Two cubics of the net associated with the complete quadrangle of a Steinerian transformation always intersect in two corresponding points. 
All cubics of the net have, of course, the seven fixed points of the complete quadrangle in common.

3. Let $P\left(a_{1}, a_{2}, a_{3}\right)$ and $Q\left(b_{1}, b_{2}, b_{3}\right)$ be the $S$-points of two cubics $C_{P}$ and $C_{Q}$ of the net, so that the equation of the pencil of cubics $C_{\lambda}$ determined by $C_{P}$ and $C_{Q}$ may be written in the form

$$
\begin{aligned}
&\left(a_{1}+\lambda b_{1}\right) x_{2} x_{3}\left(x_{2}-x_{3}\right)+\left(a_{2}+\lambda b_{2}\right) x_{3} x_{1}\left(x_{3}-x_{1}\right) \\
&+\left(a_{3}+\lambda b_{3}\right) x_{1} x_{2}\left(x_{1}-x_{2}\right)=0 .
\end{aligned}
$$

Obviously, the $S$-point of every cubic of this pencil with the parameter $\lambda$ has the coordinates

$$
a_{1}+\lambda b_{1}, \quad a_{2}+\lambda b_{2}, \quad a_{3}+\lambda b_{3},
$$

and lies on the line joining the $S$-points $P$ and $Q$ of $C_{P}$ and $C_{Q}$.

As the equation of every pencil of the net may be written in the form (3) we may state the

Theorem: The S-points of the cubics of a pencil within the net of cubics associated with a Steinerian quadruple are collinear, and form a point set projective with the pencil of cubics.

Let $l$ be the line of collinearity of the $S$-points of the pencil of cubics, and $S_{\lambda}$ the $S$-point on $l$ associated with the cubic $C_{\lambda}$ of the pencil. The cubic $C_{\lambda}$ will pass through the two corresponding points $P$ and $P^{\prime}$ of the Steinerian transformation which lie on $l$. As there are two and only two such points on $l$, it is clear that all cubics of the pencil must pass through these points. We shall call the locus $l$ of the $S$-points of the pencil the $S$-line of the pencil of cubics $C_{\lambda}$.

Consider now the pencil of cubics $C_{\lambda}$, and join every $S$ point on the $S$-line $l$ to a fixed point $S$ not on $l$. In this manner we get the pencil of lines through $S$ with the equation $\left(s_{1}, s_{2}, s_{3}\right.$ being the coordinates of $\left.S\right)$

$$
\begin{aligned}
& \left(s_{2} a_{3}-s_{3} a_{2}\right) x_{1}+\left(s_{3} a_{1}-s_{1} a_{3}\right) x_{2}+\left(s_{1} a_{2}-s_{2} a_{1}\right) x_{3} \\
& +\lambda\left\{\left(s_{2} b_{3}-s_{3} b_{2}\right) x_{1}+\left(s_{3} b_{1}-s_{1} b_{3}\right) x_{2}\right. \\
& \left.+\left(s_{1} b_{2}-s_{2} b_{1}\right) x_{3}\right\}=0
\end{aligned}
$$

which is projective with the set of $S$-points, and consequently with the pencil of cubics (3). These two projective pencils generate a quartic whose equation is found as 
(5) $\left\{\left\{\left(a_{2} b_{3}-a_{3} b_{2}\right) x_{1}+\left(a_{3} b_{1}-a_{1} b_{3}\right) x_{2}+\left(a_{1} b_{2}-a_{2} b_{1}\right) x_{3}\right\}\right.$ $\times\left\{s_{1} x_{2} x_{3}\left(x_{2}-x_{3}\right)+s_{1} x_{3} x_{1}\left(x_{3}-x_{1}\right)\right.$

$$
\left.+s_{3} x_{1} x_{2}\left(x_{1}-x_{2}\right)\right\}=0 .
$$

Clearly, the quartic degenerates into the $S$-line of the pencil of cubics, and into a cubic having $\left(s_{1}, s_{2}, s_{3}\right)$ as the $S$-point. Hence the

Theorem: Any cubic of the net with a given S-point may be generated by any pencil of cubics within the net, not containing the given cubic, and the projective pencil of lines joining the Spoint of the given cubic to the $S$-points of the cubics of the pencil.

Consider next two pencils of cubics $C_{\lambda}$ with the $S$-line $l$, and $C_{\mu}$ with the $S$-line $m$, and a point $S$, not on $l$ or $m$. Draw any line $g$ through $S$, cutting $l$ and $m$ in $S_{\lambda}$ and $S_{\mu}$, and construct the cubics $C_{\lambda}$ and $C_{\mu}$ having $S_{\lambda}$ and $S_{\mu}$ as $S$-points.

They both pass through the two fixed points $P$ and $P^{\prime}$ on $g$ corresponding to each other in the Steinerian transformation. But $P$ and $P^{\prime}$ also lie on the cubic $C_{S}$ associated with $S$ as an $S$-point. For a variable $g$ through $S, S_{\lambda}$ and $S_{\mu}$ describe two perspective point sets on $l$ and $m$ which are projective with the pencils of cubics $C_{\lambda}$ and $C_{\mu}$. These pencils are therefore themselves projective, and generate the cubic $C_{S}$. Hence the

TheOREM: Every cubic of the net associated with a Steinerian transformation may be generated in an infinite number of ways by projective pencils of cubics of the same net.

UNIVERSITY OF ILLINOIS.

\section{SOME THEOREMS OF COMPARISON AND OSCILLATION.}

BY PROFESSOR TOMLINSON FORT.

(Read before the American Mathematical Society September 4, 1917.)

Theorem I: Given

$$
\frac{d}{d x}\left(K_{1}(x) y_{1}{ }^{\prime}\right)+G_{1}(x) y_{1}=0
$$

and

$$
\frac{d}{d x}\left(K_{2}(x) y_{2}{ }^{\prime}\right)+G_{2}(x) y_{2}=0,
$$

